\title{
Article
}

\section{To focus or not to focus: Is attention on the core components of action beneficial for cycling performance?}

Bertollo, Maurizio, di Fronso, Selenia, Filho, Edson, Lamberti, Vito, Ripari, Patrizio, Machado Reis, Victor, Comani, Silvia, Bortoli, Laura and Robazza, Claudio

Available at http://clok.uclan.ac.uk/14055/

Bertollo, Maurizio, di Fronso, Selenia, Filho, Edson ORCID: 0000-0002-85484651, Lamberti, Vito, Ripari, Patrizio, Machado Reis, Victor, Comani, Silvia, Bortoli, Laura and Robazza, Claudio (2015) To focus or not to focus: Is attention on the core components of action beneficial for cycling performance? The Sport Psychologist, 29 (2). pp. 110-119. ISSN 0888-4781

It is advisable to refer to the publisher's version if you intend to cite from the work.

For more information about UCLan's research in this area go to

http://www.uclan.ac.uk/researchgroups/ and search for <name of research Group>.

For information about Research generally at UCLan please go to http://www.uclan.ac.uk/research/

All outputs in CLoK are protected by Intellectual Property Rights law, including Copyright law. Copyright, IPR and Moral Rights for the works on this site are retained by the individual authors and/or other copyright owners. Terms and conditions for use of this material are defined in the policies page. 
1

2

3

5

6

7

8

9

10

11

12

13

14

15

16

17

18

19

20

21

22

23

24

25

26

27

28

29

30

31

32

\section{To focus or not to focus: Is attention on the core components of}

\section{action beneficial for cycling performance?}

Maurizio Bertollo $^{\mathrm{a}}$, Selenia di Fronso ${ }^{\mathrm{a}}$, Edson Filho ${ }^{\mathrm{b}}$, Vito Lamberti ${ }^{\mathrm{a}}$, Patrizio Ripari ${ }^{\mathrm{a}}$, Victor

Machado Reis ${ }^{\mathrm{c}}$, Silvia Comani ${ }^{\mathrm{a}}$, Laura Bortoli ${ }^{\mathrm{a}} \&$ Claudio Robazza $^{\mathrm{a}}$

a University "G. d’Annunzio” of Chieti-Pescara

${ }^{b}$ University of Central Lancashire

${ }^{\text {c }}$ University of Tras-os-Montes and Alto Douro

(1)

Corresponding Author:

Maurizio Bertolli

BIND - Behavioral Imaging and Neural Dynamics Center

Dept. of Neuroscience, Imaging and Clinical Sciences

University "G. d'Annunzio" of Chieti-Pescara

Via dei Vestini, 33

66013 Chieti, Italy

m.bertollo@unich.it 
2 We conducted a counterbalanced repeated measure trial to investigate the effect of different

3 internal and external associative strategies on endurance performance. Seventeen college-

4 aged students were randomly assigned to three experimental conditions to test the notion that

5 different attention-performance types (optimal Type 1, functional Type 2, and dysfunctional

6 Type 3) would influence endurance time on a cycling task. Specifically, Type 1 represented

7 an effortless and automatic, "flow-feeling” attentional mode. Type 2 referred to an

8 associative focus directed at core components of the task. Type 3 represented an attentional

9 focus directed at irrelevant components of the task. Participants completed three time-to-

10 exhaustion-tests while reporting their perceived exertion and affective states (arousal and

11 hedonic tone). Results revealed that Type 1 and Type 2 attentional strategies, compared to

12 Type 3 strategy, exerted functional effects on performance, whereas a Type 3 strategy was

13 linked to lower performance, and lower levels of arousal and pleasantness. Applied

14 implications are discussed.

16 Keywords: Attentional focus, cycling, fatigue, endurance, multi-action plan model. 
To Focus or not to Focus: Is Attention on the Core Components of Action Beneficial for Cycling Performance?

There is general agreement about the importance of studying how different attentional strategies influence performance in sport and exercise settings (Basevitch et al., 2011; Blanchfield, Hardy, de Morree, Staiano, \& Marcora, 2014; Connolly \& Tenenbaum, 2010; Hutchinson \& Tenenbaum, 2007; Razon et al., 2010; for a review, see Brick, MacIntyre, \& Campbell 2014). In this regard, previous research has shown that one’s ability to self-regulate attentional focus (e.g., attentional flexibility) is associated with the ability to sustain exertive effort in endurance tasks (for a review, see Tenenbaum, 2005). To perform optimally, athletes must be able to employ different attentional strategies in order to control external and internal distracters, while focusing on body and task-relevant cues (Tenenbaum, 2001, 2005).

\section{Attentional Strategies for Endurance Performance}

Early research suggested that there are primarily two coping strategies that can be used to enhance performance in endurance tasks (a) “association” and (b) “dissociation” (Weinberg, Smith, Jackson, \& Gould, 1984). Association occurs when people monitor their body sensations (e.g., respiration rate, body temperature, muscle pain and tightness), while reminding themselves to relax and modify stride and pace to secure greater running economy. Indeed, elite performers monitor their body sensations more effectively than their less accomplished counterparts (Raglin \& Wilson, 2008). Dissociation occurs when individuals ignore pain, fatigue, or boredom by directing their attention outwards or by focusing on pleasant stimuli (e.g., listening to music; Weinberg et al., 1984). In this regard, Schücker and colleagues found that external attentional focus has a significant impact on physiological performance measures of running economy (Schücker, Anheier, Hagemann, Strauss, \& Völker, 2013). 
Association and dissociation are examples of attention control, which is a topic of great interest to sport and exercise psychologists. In essence, associative and dissociative strategies represent two distinct cognitive styles that indicate where individuals allocate attention to improve adjustment to a physical task (Tenenbaum, 2005). This initial distinction between the two broad categories of attention focus (as association and dissociation) was introduced by Morgan and Pollock (1977), and has since oriented research on attentional focus and physical effort (Hutchinson \& Tenenbaum, 2007; Stanley, Pargman, \& Tenenbaum, 2007; Stevinson \& Biddle, 1998). According to Morgan and Pollock, association is an internal attentional style used by people to monitor sensorial input while performing a physical task. Dissociation pertains to any cognitive strategy used to divert attention away from internal sensations and toward external distractions. Schomer (1986) differentiated associative and dissociative strategies by discussing the presence of "task-related" and "taskunrelated” thoughts. Specifically, associative thoughts are related to the task at hand (e.g., bodily sensations, performance instructions, and pace monitoring), while dissociative thoughts are not relevant to the task (e.g., reflective activity thoughts, problem solving). Stevinson and Biddle (1998) argued that a dichotomous treatment of attentional focus was inherently simplistic, and therefore proposed a two dimensional model considering: (a) the direction of attention (internal or external), and (b) task relevance (relevant or irrelevant). Internal strategies allow an individual to monitor his/her internal states while making appropriate psychophysiological adjustments to accommodate pain and effort. Conversely, external strategies allow the performer to shift attention to exterior events, thus reducing perceptions of exertion. Task-relevant thoughts involving an internal focus (e.g., physical sensations) are classified as internal association, whereas task-relevant thoughts with an external focus (e.g., pacing) are labeled external association. Similarly, task irrelevant thoughts with an internal focus (e.g., daydreams) were categorized as internal dissociation 
1 and task-irrelevant thoughts with an external focus (e.g., scenery) as external dissociation.

2 Drawing on Stevinson and Biddle’s (1998) classification, Brick et al. (2014) suggested that

3 the associative dimension should also include active self-regulation (i.e., thoughts related to

4 cadence, pacing, technique, strategy, or maintaining a relaxed state) and internal sensory

5 monitoring.

6

Tenenbaum's (2005) effort-related model added the notion that associative and dissociative focus depends on workload intensity. In particular, Tenenbaum observed that people may intentionally switch their attentional focus, between associative and dissociative strategies, under low workload intensities. However, when physical symptoms of exertion reach a threshold upon which attention flexibility (i.e., ease of switching back and forth between dissociative to an associative pattern) is compromised, a final switch from a dissociative to an associative focus occurs (e.g., increased somatic awareness, and pain; see Connolly \& Tenenbaum, 2010; Hutchinson \& Tenenbaum, 2007; Stanley et al., 2007). In this regard, endurance athletes have reported that focusing on internal (association) cues is functional for performance unless they feel very tired and distressed, when an associative strategy is viewed as dysfunctional and unnatural (Masters \& Ogles, 1998).

It is important to note that research on the relationship between performance and attentional focus (as associative or dissociative) has produced conflicting results. While some scholars have found a linkage between higher ratings of perceived exertion (RPE) and an associative focus (Baden, McLean, Tucker, Noakes, \& Gibson, 2005), others observed a linkage between higher RPE and a dissociative focus (Beaudoin, Crews, \& Morgan, 1998; Brewer, Van Raalte, \& Linder, 1996). Furthermore, there is also literature suggesting no differences in performance as a function of attentional focus (Harte \& Eifert, 1995; Weinberg et al., 1984). Despite these conflicting results, there is a general agreement that the optimization of attentional control may produce significant gains in endurance performance 
1 and external focus strategies can also be beneficial to performance beside the internal ones

2 (Schücker et al., 2013). The purpose herein was to investigate the effect of different internal and external associative strategies on endurance performance in cycling, using the multiaction plan (MAP) model as a theoretical basis for our experimental protocol.

\section{The Multi-Action Plan Model}

The recently proposed MAP model is based on the notion that different attentional strategies lead to different performance states, namely optimal and less than optimal (Bertollo, Bortoli, Gramaccioni, Hanin, Comani, \& Robazza, 2013; Bortoli, Bertollo, Hanin, \& Robazza, 2012). Specifically, an automatic attentional focus (Type 1) has been linked to optimal performance in sports. However, an attentional focus directed at a core component of a given action (Type 2) has also been associated with functional performance, which is defined within the individual zones of optimal functioning (IZOF) framework as an individual's effective recruitment and use of available resources for optimal achievements (Hanin, 2007). In contrast, over-controlled attentional focus has been found to lead to dysfunctional performance in sports. Previous research based on the MAP model revealed that attentional focus moderates performance quality in self-paced tasks (i.e., rifle and pistol shooting). In effect, Bortoli et al. (2012) observed that four performance categories result from different attentional strategies. These performance states are: (a) Type 1, optimal performance, characterized by an automatic (“flow-feeling” like) attentional mode and pleasant-functional emotions; (b) Type 2, functional performance, typified by an associative focus directed at core components of a given task/action and pleasant or unpleasantfunctional emotions; (c) Type 3, dysfunctional performance, characterized by a focus directed at irrelevant components of a given task/action and unpleasant-dysfunctional emotions; and (d) Type 4, poor performance, typified by a markedly irrelevant focus and pleasantdysfunctional emotions. 
The MAP model was developed to orient applied interventions aimed at reaching and maintaining maximal performance in presence of distress, fatigue, and distracting situations. In detail, the MAP model is conceptualized as a function of distinct performance levels (i.e., optimal or suboptimal) and attentional demands (i.e., automatic or controlled), thus establishing four performance categories: Optimal-automatic (Type 1), optimal-controlled (Type 2), suboptimal-controlled (Type 3), and suboptimal-automatic (Type 4). Indeed, these four types of performance have been found to rely on specific psychophysiological patterns, including skin conductance levels, respiration rate, and fronto-occipital and inter-frontal coherence in the alpha band (Bertollo et al., 2013; Comani et al., 2014). Of note, perceived arousal and pleasantness levels have also been found to predict performance in endurance tasks in general (Hanin, 2007), and in respect to the MAP model’s performance categorization in particular (Bertollo et al., 2013). In fact, arousal and pleasantness underlie the notion of core affect, thus influencing one’s ability to perform a given task (Russell\& Weeks, 1994; Russell, Weiss, \& Mendelsohn, 1989). Hence, in the present study, we were also interested in assessing whether the cyclists' core affect (i.e., arousal and hedonic tone) patterns would differ in regards to the MAP model categories.

It is also important to note that the MAP model is idiosyncratic in nature, thus assuming that ones' strategies and behaviors during performance are unique. In essence, the MAP model posits that individuals who focus on their idiosyncratic core components of action in conditions of distress or fatigue are likely to consistently attain high performance levels. In a recent study, for instance, cyclists identified "pedaling rate” as a core component of action linked to endurance performance (Comani et al., 2014). To this extent, Bortoli et al. (2012) suggested that an appropriate focus on one or a few core components of the action helps performers to self-regulate by maintaining optimal action tendencies. In contrast, reinvesting attention on body feelings (e.g., muscular tension, muscular stiffness, and pain) in 
1 the attempt to control the whole action tends to increase the likelihood of performance

2 breakdown (Masters \& Maxwell, 2008). According to the MAP model, individuals can reach

3 functional performance levels by directing their attention on the core components of the

4 action (i.e., using action-centered strategies) and/or optimizing their emotional states (i.e.,

5 using emotion-focused strategies).

\section{The Present Study}

We investigated the effect of different internal and external associative strategies on endurance performance. Drawing on the MAP model assumptions, we hypothesized that participants in a Type 1 performance condition would experience optimal performance and a “flow state” typified by pleasant affect, while externally concentrating on pacing. Type 2 performance situation, in which participants' attention was directed internally on the core component of the cycling action, was expected to result in a functional state and be accompanied by pleasant or unpleasant affect. Finally, Type 3 performance condition was predicted to augment individual's fatigue sensations and cause a suboptimal performance state because of excessive focus on muscle feelings and pacing. Type 4 performance, characterized by unfocused attention and poor performance, was not considered in the current study because it is irrelevant to the development of applied guidelines for performance improvement in sports.

\section{Method}

\section{Design}

Based on the performance states delineated in the MAP model, we conducted a counterbalanced repeated measure trial to investigate the effect of different internal and external associative strategies on endurance performance. This is congruent with the importance of testing the MAP model assumptions in sport modalities other than self-paced sports (e.g., dart throwing, pistol shooting), especially endurance sports in which attentional 
1 control and performance are intrinsically related. In particular, we tested participants in time-

2 to-exhaustion trials during cycling, while collecting psychological markers of fatigue and

3 affect (i.e., RPE, hedonic tone and arousal).

$4 \quad$ Participants

5

A priori power analysis (effect size $=.50$, power of .95 , and an alpha level of .05 ) was used to determine the sample size $(N=18)$. In order to detect a moderate effect size (see Cohen, 1988), we recruited 21 college-aged students. Four students discontinued participation from the experiment due to either personal or health reasons. Accordingly, seventeen students (5 women and 12 men, $M_{\text {age }}=24.3$ years, $S D=4.9$ years) completed the experimental protocol, consisting of four visits to an exercise physiology laboratory. All seventeen volunteers participated regularly in different physical activities of low or moderate intensity. Assessment at baseline revealed that the fitness level of participants was generally low (men: $\dot{\mathrm{V}} \mathrm{O}_{2} \mathrm{max} \mathrm{M}=39.03 \mathrm{~mL} \mathrm{~kg}^{-1} \mathrm{~m}^{-1}, S D=15.64$, power peak output $M=231.54, S D$ = 69.01; women: $\dot{\mathrm{V}}{ }_{2} \max M=26.31 \mathrm{~mL} \mathrm{~kg}^{-1} \mathrm{~m}^{-1} S D=5.32$, power peak output $M=118.57$, $S D=13.91)$. After being briefed on the general purpose of the study, the participants agreed to participate and signed a written informed consent. The study was conducted in accordance with the declaration of Helsinki and received approval from the local university ethics committee.

\section{Measurements}

Ratings of Perceived Exertion (RPE). RPE was measured through a CR-10 Scale ${ }^{\circledR}$ ranging from “0” (no effort) to “•” (maximal sustainable effort). The verbal anchors were: 0 = nothing at all, $0.5=$ extremely weak, $1=$ very weak, $2=$ weak, $3=$ moderate, $5=$ strong, 7 $=$ very strong, 10 = extremely strong, $\bullet=$ absolute maximum. No verbal anchors were used for 4, 6, 8 and 9. Of note, the CR-10 Scale ${ }^{\circledR}$ is instrumental in diminishing ceiling effects as 
1 its ratings are linearly related to various physiological parameters such as $\dot{\mathrm{V}} \mathrm{O}_{2}$ max, lactate, 2 and heart rate (Borg, 1998).

Affect grid. This is a single-item scale designed to quickly assess core affect along the dimensions of pleasure-displeasure and sleepiness-arousal (Russell et al., 1989). In our study, the participants were asked to place a single "X mark" on the 9x9 grid, which columns represent pleasantness and arousal scores. Hence, both the pleasure-displeasure and sleepiness-arousal can range from 1 to 9.

Manipulation check questionnaire. The participants were asked to rate, using a 10point frequency scale with anchors 1 (never) and 10 (always), one of the following questions: "How often did you focus your attention on the metronome?” (Type 1 performance condition), "How often did you focus your attention on your feet to maintain individual RPM pacing?” (Type 2 performance condition), and “How often did you focus your attention on the tension of your muscles and body or fatigue?” (Type 3 performance condition).

\section{Procedures}

Four visits to the laboratory were planned, with inter-visit intervals of 48 to 72 hours. Two trained scholars collected the data. Data collection occurred in a quiet (no music playing and no other people allowed in the laboratory) and safe environment to ensure the comfort of the participants. During the first session, the cycle ergometer was set-up and adjusted to each participant's needs. The participants used the same cycle ergometer set-up during the subsequent visits. They were allowed to ask questions at any time during the study.

Incremental test. During the first visit to the laboratory, participants received standard instructions about the use of the Borg CR-10 RPE scale (Borg \& Borg, 2001) and the affect grid (Russell et al., 1989). They also performed an incremental test to determine their anaerobic threshold or second ventilatory threshold (VT2). Specifically, after a warm-up (4 min cycling at 25 watt), $\dot{\mathrm{VO}} 2$ and $\square \mathrm{CO}_{2}$ were measured using an incremental protocol on 
1 a Monark Cycle Ergometer (939 E). Heart rate, $\square \mathrm{O}_{2}$ and $\square \mathrm{CO}_{2}$ were continuously monitored

2 during the exercise using a Schiller CS 200 system. VT2 was determined through the V-

3 Slope method (Wasserman, Stringer, Casaburi, Koike, \& Cooper, 1994). Pedal rate was set at

470 revolutions per minute (rpm) and the workload power output was initially set at $25 \mathrm{~W}$.

5 Subsequently, the power output was incrementally increased by $25 \mathrm{~W}$ every 2 min until

6 exhaustion. After the completion of the incremental test, the participants were given a 20 min

7 rest period. After this period, participants were asked to pedal at VT2+5\% for ten minutes in

8 order to identify their preferred pedaling rate (PPR), while familiarizing themselves with the

9 study's procedures. Overall, this initial assessment indicated that participants were not trained athletes but rather recreational exercisers and, in some instances, arguably unfit individuals. Lastly, the participants were assigned to three different experimental conditions to be undertaken separately during the subsequent three meetings. These conditions required that the participants kept their focus of attention on either: (a) a metronome that reproduced their PPR (external associative strategy) aimed at eliciting a Type 1 performance state, which is typified by movement automaticity and optimal-pleasant affect; (b) their PPR (i.e., internal associative strategy on pacing representing the core component of action) aimed at eliciting a Type 2 performance condition typified by focused attention on the relevant aspects of action and pleasant or unpleasant affect; or (c) muscle fatigue feelings and difficulties in maintaining pacing (dysfunctional associative strategy on internal feelings) aimed at inducing Type 3 dysfunctional performance state and unpleasant affect (see Figure 1).

Time-to-exhaustion test at individual constant load. During the subsequent visits to the lab, participants were assigned to one of the three experimental conditions, each defined in a random order and occurring on different days. They performed a time-to-exhaustion test, at individual VT2 power intensity, while reporting their RPE and affective states. Time-toexhaustion was determined as either (a) the maximum interval in which the participants could 
1 maintain exercise intensity (VT2 + 5\%), and/or (b) the moment in which participants'

2 reached volitional exhaustion. The first criterion, in particular, was established to prevent excessive lengthening of the experimental condition, especially with well-trained individuals.

Individual VT2 and PPR of each participant was set during test. After a resting period (no movement) of $2 \mathrm{~min}$, and a warm-up period of $4 \mathrm{~min}$ on the cycle ergometer at individual power (calculated as the $40-50 \%$ of the individual $\dot{\mathrm{V}}_{2} \max$ ), the participants performed a constant load until exhaustion at their individual power (i.e., VT2 + 5\% with a PPR) while maintaining their PPR. After exhaustion, the participants engaged in an active recovery period of 4 min (at the same power used during warm-up) followed by a resting period of 2 min (no movement). RPE and affect grid scores were collected in the last $5 \mathrm{~s}$ of every 1 min period throughout the entire test. The manipulation check questionnaire was administered at the end of data acquisition to verify adherence to the experimental conditions.

\section{Statistical Analysis}

Analysis of variance with repeated measures (RM-ANOVA), with an alpha level set at .05, were computed to compare participants' time-to-exhaustion test, RPE, and affective scores across the three experimental conditions. Bonferroni post-hoc test was used to identify potential differences among the three experimental conditions. Furthermore, condition $\times$ time RM-ANOVAs were performed for RPE and affect grid scores at 0\% (first minute), 25\%, 50\%, 75\%, and 100\% (last completed minute) in the time-to-exhaustion test. This is aligned with the importance of measuring temporal changes in affective states in general (Hanin, 2007), and in fatigue in particular (Blanchfield et al., 2014). These iso-times were measured at the selected time-points, thus allowing for the identification of affective (i.e., arousal and pleasantness) and perceptual (RPE) changes throughout the exhaustion tests. Specifically, iso-time values for $0 \%$ corresponded to the values for the first full minute of each time-toexhaustion test. The value of iso-time at $100 \%$ was defined as the shortest time-to-exhaustion 
1 accomplished by each individual over their three tests. The minute $100 \%$ iso-time was divided by two to obtain the value corresponding to 50\% iso-time (see Blanchfield et al., 2014). The $25 \%$ and $75 \%$ iso-times were derived accordingly.

\section{Results}

\section{Manipulation Check}

Manipulation check results showed that participants adhered satisfactorily to the experimental conditions. During the Type 1 performance condition, response ratings ranged from 5 to 9, which corresponded to an adherence frequency from often to almost always $(M=$ 6.82, $S D=.87)$. In the Type 2 performance state, the response ratings ranged from 6 to 9 (very often to almost always; $M=7.29, S D=.89$ ), whereas in the Type 3 performance state the response ratings ranged from 7 to $9(M=7.82, S D=.74)$. The levels of arousal, affect, and perception of effort were also analyzed prior to the time-to-exhaustion test. RM-ANOVA results on the affect grid and RPE data collected during the rest and warm-up phases revealed no differences among the experimental conditions before the time-to-exhaustion test in regards to arousal level, $F(2,32)=0.89, p=.41$, hedonic tone, $F(2,32)=2.32, p=.11$, and RPE, $F(2,32)=0.76, p=.48$.

\section{Experimental Manipulation}

Descriptive analysis. Descriptive statistics for all measures are reported in Table 1.

High standard deviation scores on time-to-exhaustion indicate large individual differences in the efficacy of the Type 1 and Type 2 strategies. Two examples of idiosyncratic trends for RPE during the entire experimental phase are presented in Figure 2. Panel A shows the RPE trend of a cyclist who reached best performance using Type 1 performance strategy. On the other hand, panel B shows the RPE trend of a cyclist who sustained a longer time in cycling performance through Type 2 performance strategy. Both participants showed poorer performance under the Type 3 performance condition. 
Inferential analysis. In RM-ANOVA, the assumption of sphericity was violated, and thus the Greenhouse-Geisser correction was applied to the degrees of freedom for subsequent F statistic calculation. RM-ANOVA on overall scores showed differences across the three

experimental conditions in regard to the duration of the time-to-exhaustion test, $F(1.81$, 29.03) $=11.41, p<.01, \eta_{\mathrm{p}}{ }^{2}=.41$, power .98. Bonferroni post-hoc test showed differences in the duration of time-to-exhaustion test between Type $1(M=18.35 \mathrm{~min})$ and Type $3(M=$ $14.12 \mathrm{~min})$ performance states $(p<.01)$, and between Type $2(M=17.65 \mathrm{~min})$ and Type $3(M$ $=14.12 \mathrm{~min})$ performance states $(p<.01)$.

To explore the effect of internal and external associative strategies on RPE, based on the MAP model assumptions, we calculated the slope of RPE for the time-to-exhaustion test. Subsequently, we performed a RM-ANOVA on the overall slope scores to explore the impact of RPE, and affect trend during task. Results revealed differences among the three experimental conditions on the slope scores of RPE during time-to-exhaustion-test, $F(1.67$, 26.86) $=4.01, p=.03, \eta_{\mathrm{p}}{ }^{2}=.21$, power .62. In particular, Bonferroni post-hoc test showed difference between Type $1(M=0.67)$ and Type $3(M=0.88)$ performance states $(p<.01)$ and Type $2(M=0.73)$ and Type $3(M=0.88)$ performance states $(p=.04)$. No differences were found with regards to the RPE slope during the recovery period, and scores of arousal and hedonic tone.

Condition $\times$ iso-time RM-ANOVAs on RPE, arousal, and hedonic tone were also performed. Specifically, we compared the three experimental conditions over iso-time for RPE. Results revealed an effect on condition, $F(1.89,30.24)=462.46, p<.01, \eta_{\mathrm{p}}{ }^{2}=.97$, power 1.00, on time, $F(2.32,37.13)=74.29, p<.01, \eta_{\mathrm{p}}{ }^{2}=.82$, power 1.00 , and in the interaction between condition and time, $F(2.78,44.53)=6.57, p=.01, \eta_{\mathrm{p}}{ }^{2}=.29$, power .95 . These differences are graphically depicted in Figure 3 (Panel A), in which higher RPE levels are more evident for Type 3 performance state at the $75 \%$ and $100 \%$ iso-times. 
Furthermore, when comparing the three experimental conditions over iso-time on

2 Arousal level, we found an effect on condition, $F(1.34,21.55)=10.47, p<.01, \eta_{\mathrm{p}}{ }^{2}=.39$,

3 power .93, time, $F(3.11,39.49)=3.80, p<.01, \eta_{\mathrm{p}}{ }^{2}=.34$, power .99 , and in the interaction

4 between condition and time, $F(3.47,55.60)=6.57, p<.01 \eta_{\mathrm{p}}{ }^{2}=.19$, power .83 . These

5 differences can be seen in Figure 3 (Panel B), in which lower levels of arousal accompanied

6 Type 3 performance throughout the entire test. Finally, significant differences were observed

7 for hedonic tone on condition, $F(1.55,24.80)=21.64, p<.01, \eta_{\mathrm{p}}^{2}=.57$, power 1.00 , and

8 time, $F(2.52,40.85)=4.92, p<.01, \eta_{\mathrm{p}}{ }^{2}=.23$, power .84 , but not for the interaction (Figure 3, 9 Panel C).

\section{Discussion}

We conducted a counterbalanced repeated measure trial to investigate the effect of different internal and external associative strategies on endurance performance. Based on the MAP model, we differentiated among an external focus associated with a fluent performance (Type 1), an internal focus linked to optimal performance regulation (Type 2), and an internal focus hindering automatic execution of motor performance (Type 3). Results reinforced the notion that fatigue is a natural psychobiological process related to attentional focus and motivation (Blanchfield et al., 2014; Schücker et al., 2013). Specifically, both external (Type 1) and internal (Type 2) associative attention strategies, in comparison with internal attentional focus (Type 3), were found to be functional, whereas intense internal attentional focus (Type 3) was found to be dysfunctional to performance. Of note, the functional effects of Type 1 and Type 2 performance states observed herein have also been identified in shooters (Bertollo et al. 2013; Bortoli et al., 2012). Furthermore, findings pertaining to Type 3 performance states are consistent with the reinvestment hypothesis, in which automatic movements are disrupted when individuals try to consciously monitor and over control both 
1 feedback input (e.g., feelings of fatigue) and movement execution (Masters \& Maxwell, 2 2008).

Our results showed that cyclists were able to reach and maintain optimal performance when using an external associative strategy by focusing attention on metronome (Type 1 performance). Similarly, the cyclists were able to perform optimally when using an internal associative strategy with attention focused on the core component of the action (i.e., PPR, Type 2 performance). However, when the cyclists focused their attention on internal and irrelevant features of the task (e.g., disruption of PPR or muscle tension), they performed poorly (dysfunctional performance). These results suggest that in the absence of flow-like performance states, individuals may still perform well by adopting a Type 2 strategy. Therefore, applied sport and exercise psychologists should assist individuals in identifying and focusing on their (idiosyncratic) core components of action linked to functional performance patterns. In essence, by learning how to focus on core components of action individuals may be able to perform well while ignoring unpleasant feelings of fatigue (e.g., muscle pain).

Our findings pertaining to Type 2 performance are in agreement with the notion that a functional internal focus (internal association) may also lead to functional performance experiences. To this extent, Masters and Ogles (1998) noted that an internal associative strategy is neither dysfunctional nor unnatural. Accordingly, individuals may benefit from internal associative strategies, especially under high workload intensities when the ability to switch between association and dissociation is compromised (Tenenbaum, 2005). In this regard, it is important to note large interindividual differences among the cyclists, with some individuals being remarkably unfit/sedentary. The differences were in the intensity, variability, and magnitude of the cyclists' subjective and psychophysiological recordings. Overall, these results are in accordance with the individuality principle and reflect the 
1 idiosyncratic nature of maximal performance in sports (Hanin, 2007). In effect, some

2 individuals may perform better using external strategies while others experience maximal

3 endurance performance when adhering to internal strategies (Bortoli et al., 2012). In fact,

4 although applying different methodological approaches various scholars have emphasized the

5 importance of identifying individual difference of performance-related states (Bortoli et al.,

6 2012; Filho, Moraes, \& Tenenbaum, 2008). There is also a general agreement on the

7 importance of identifying the fundamental variables and mechanisms linked to peak

8 performance in sports (Hanin, 2007).

Although internal strategies may be functional from an idiosyncratic standpoint, our nomothetic (group level) analysis echoed the notion that (a) external attentional focus is best for performance gains in economy of effort (Schücker et al., 2013), and (b) associativedissociative dimension is the main determinant of RPE (Stanley et al., 2007). In this regard, exergaming technology can be used to create “dissociative environments” (e.g., gym, physical therapy clinics) aimed at diverting attention from feelings of fatigue. In addition to gaming technology, sport and exercise psychologists may use bio-neurofeedback multimedia modalities to help people to divert attention away from unpleasant fatigue sensations (Perry, 2012).

We also measured core affect throughout the time-to-exhaustion-tests. The results revealed that affective value means (arousal and hedonic tone) did not differ across conditions. However, when comparing iso-times across conditions, we observed differences for both arousal and pleasantness trends. Thus, the relationship between iso-time and RPE was found to be influenced by attentional strategies, with an internal dysfunctional associative focus leading to lower levels of arousal and pleasantness. The fact that Type 3 performance was associated with lower levels of pleasure and arousal, suggests that focusing on fatigue feelings is related to energy demobilization. Indeed, Hanin (2007) posited that 
1 optimal performance is likely to occur when energy matches task demands (i.e., energy

2 matching hypothesis). The flow-feeling theory also reflects the notion that optimal

3 performance is likely to occur when one's psychosocial skills are "a good match" for a given

4 challenging task (Csikszentmihalyi \& Csikszentmihalyi, 1993). It is also important to note

5 that individuals have different arousal and hedonic tone levels linked to optimal and less than

6 optimal performance (Robazza, Pellizzari, Bertollo, \& Hanin, 2008). In this regard, Hanin

7 (2007) has noted that there is interindividual variability in the intensity and content of

8 idiosyncratic functional and dysfunctional affective states.

Overall, three main conclusions derive from our study: (a) both internal and external attention strategies, namely Type 1 and 2 performance states, can exert functional effects on performance compared to attentional focus on feelings of fatigue (Type 3 performance); (b) internal attention can be functional if the attentional focus is directed toward the core component of action (Type 2 performance) rather than on feelings of fatigue (Type 3 performance); and (c) attentional focus on feelings of muscle fatigue leads to poor performance and low pleasant states and arousal levels (thereby causing energy demobilization) during high intensity exercise (iso-time $>50 \%$ ). These conclusions are congruent with the MAP model performance categorization, particularly with the notion that individuals can perform well when directing their attentional focus to the core components of action related to a given exertive task. From a broader theoretical standpoint, these findings support a top down psychobiological account of endurance fatigue (Marcora, 2009) in which exhaustion is viewed as a volitional choice influenced by psychological factors (e.g., attentional and motivational strategies) rather than a process determined by afferent feedback from the muscular and cardiovascular systems. In fact, this new psychobiological model has been seen as an alternative to the tradition peripheral afferent feedback framework (i.e., inhibitory feedback triggered by increased concentration of metabolites such as lactate and 
urea; Gandevia, 2001) in trying to explain exhaustion in humans (Blanchfield et al., 2014; Marcora, 2009).

It is also important to acknowledge the limitations of our study in attempt to better orient future research efforts. First, it is difficult to induce Type 1, flow-like performance in both laboratory and ecological settings (Csikszentmihalyi \& Csikszentmihalyi, 1993; Hanin, 2007). Peak performance experiences are rare, and thus pose a challenge to scholars and practitioners interested in its antecedents and outcomes. Longitudinal assessment may be an alternative to this limitation, as it allows for the recording of a larger data set with a correspondingly larger sample of peak-performance records (Filho et al., 2008). The use of a mixed-method approach, involving the measures of core affect used in this study and surveys on flow and qualitative assessments, may allow the researcher to triangulate participants' perceived flow states with bio- and neuro-feedback data, thus yielding a more comprehensive assessment of peak performance experiences in laboratory settings. A second limitation of our study is related to our convenience sample, which was comprised primarily of college students and novices. In this regard, caution should be taken in generalizing the present findings across endurance sports (e.g., road cyclists), given that participants of this study were mostly sedentary individuals. Comparing sedentary individuals with elite athletes (i.e., the expert-novice paradigm), and elite athletes among themselves (i.e., the expert performance approach) may reveal the nomological network pertaining to the attentionperformance linkage in endurance and motor tasks. Third, it could be argued that the lack of a control group may limit the generalizability of our findings. However, we opted by a counterbalanced design because a true neutral effect is somewhat unrealistic in sport and exercise psychology contexts. In this regard, extant empirical evidence suggests that attention flexibility is ultimately compromised in time-to-exhaustion trials (for a review see Tenenbaum, 2005). Notwithstanding, future studies could consider alternative experimental 
1 protocols to advance research on exertion. Finally, although a manipulation check confirmed

2 the validity of our experimental protocol, the participants did not receive training on attention

3 control to reach and maintain Type 1 and Type 2 performance states. Hence, the participants'

4 ability to adeptly control their attentional focus might not have been ideal. Future research

5 should test the effect of systematized mental skills training on individuals' ability to increase

6 the likelihood of optimal-automatic performance (Type 1), focus on their core components of

7 action (Type 2), and prevent dysfunctional performance states (Type 3).

9 processes and skills (e.g., imagery, self-talk, and goal setting) are also warranted. Qualitative

10 retrospective reports are important in the study of experts' mental processes, including meta-

11 emotional and meta-cognitive fatigue and performance experiences (Hanin, 2007). Moreover,

12 the implementation of the IZOF probabilistic methodology may generate more information

13 regarding the idiosyncratic nature of optimal performance states, as previously demonstrated

14 in other studies (e.g., Bertollo et al., 2012; Filho et al., 2008). Studying perceived exertion

15 through multi-sensorial approaches (e.g., audio and scent) may continue to advance our

16 understanding of the mechanisms underpinning fatigue in humans. 
References

Baden, D. A., McLean, T. L., Tucker, R., Noakes, T. D., \& Gibson, A. (2005). Effect of anticipation during unknown or unexpected exercise duration on rating of perceived exertion, affect, and physiological function. British Journal of Sports Medicine, 39, 742-746.

Basevitch, I., Thompson, B., Braun, R., Razon, S., Arsal, G., Tokac, U., . . Tenenbaum, G. (2011). Olfactory effects on attention allocation and perception of exertion. The Sport Psychologist, 25, 144-158.

Beaudoin, C. M., Crews, D. J., \& Morgan, D. W. (1998). Influence of psychogenic factors during a prolonged maximal run. Journal of Sport Behaviour, 21, 377-381.

Bertollo, M., Bortoli, L., Gramaccioni, G., Hanin, Y., Comani, S., \& Robazza, C. (2013). Behavioural and psychophysiological correlates of athletic performance: A test of the multi-action plan model. Applied Psychophysiology and Biofeedback, 38, 91-99. doi:10.1007/s10484-013-9211-z.

Bertollo, M., Robazza, C., Falasca, W. N., Stocchi, M., Babiloni, C., Del Percio, C., \& Comani, S. (2012). Temporal pattern of pre-shooting psycho-physiological states in elite athletes: A probabilistic approach. Psychology of Sport and Exercise, 13, 91-98, doi:10.1016/j.psychsport.2011.09.005

Blanchfield, A. W., Hardy, J., de Morree, H. M., Staiano, W., \& Marcora, S. M. (2014). Talking yourself out of exhaustion: The effects of self-talk on endurance performance. Medicine and Science in Sports and Exercise, 46, 998-1007. doi:10.1249/MSS.0000000000000184.

Borg, G. (1998). Borg's perceived exertion and pain scales. Champaign, IL: Human Kinetics. Borg, G., \& Borg, E. (2001). A new generation of scaling methods: Level-anchored ratio scaling. Psychologica, 28, 15-45. 
1 Bortoli, L., Bertollo, M., Hanin, Y., \& Robazza, C. (2012). Striving for excellence: A multiaction plan intervention model for shooters. Psychology of Sport and Exercise, 13, 693-701. doi:10.1016/j.psychsport.2012.04.006.

Brewer, B. W., Van Raalte, J. L., \& Linder D. L. (1996). Attentional focus and endurance performance. Applied Research in Coaching and Athletic Annual, 11, 1-14.

Brick, N., MacIntyre, T., \& Campbell, M. (2014). Attentional focus in endurance activity: New paradigms and future directions. International Review of Sport and Exercise Psychology, 7, 106-134. doi:10.1080/1750984X.2014.885554

Cohen, J. (1988). Statistical power analysis for the behavioral sciences (2nd ed.). Hillsdale, NJ: Lawrence Erlbaum Associates.

Comani, S., Di Fronso, S., Filho, E., Castronovo, A. M., Schmid, M., Bortoli, L., ...Bertollo, M. (2014). Attentional focus and functional connectivity in cycling: An EEG case study. International Federation for Medical and Biological Engineering, 41, 131-144.

Connolly, C. T., \& Tenenbaum, G. (2010). Exertion-attention-flow linkage under different workloads. Journal of Applied Social Psychology, 40, 1123-1145. doi:10.1111/j.15591816.2010.00613.x.

Csikszentmihalyi, M., \& Csikszentmihalyi, I. S. (1993). Family influences on the development of giftedness. In I. Selega (Ed.), The origins and development of high ability (pp. 187-206). Oxford, UK: John Wiley \& Sons.

Filho, E., Moraes, L. C., \& Tenenbaum, G. (2008). Affective and physiological states during archery competitions: Adopting and enhancing the probabilistic methodology of individual affect-related performance zones (IAPZs). Journal of Applied Sport Psychology, 20, 441-456. doi:10.1080/10413200802245221

Gandevia, S. C. (2001). Spinal and supraspinal factors in human muscle fatigue. Physiological Review, 81, 1725-1789. 
1 Hanin, Y. L. (2007). Emotions in sport: Current issues and perspectives. In G. Tenenbaum, \&

R. C. Eklund (Eds.), Handbook of sport psychology (3rd ed., pp. 31-58). New York: Wiley \& Sons.

Hargreaves, M. (2008). Fatigue mechanisms determining exercise performance: Integrative physiology is systems biology. Journal of Applied Physiology, 104, 1541-1542. doi:10.1152/japplphysiol.00088.2008.

Harte, J. L., \& Eifert, G. H. (1995). The effects of running, environment and attentional focus on athlete's catecholamine and cortisol levels and mood. Psychophysiology, 32, 4954. doi:10.1111/j.1469-8986.1995.tb03405.x.

Hutchinson, J. C., \& Tenenbaum, G. (2007). Attention focus during physical effort: The mediating role of task intensity. Psychology of Sport and Exercise, 8, 233-245. doi:10.1016/j.psychsport.2006.03.006.

Marcora, S. M. (2009). Perception of effort during exercise is independent of afferent feedback from skeletal muscles, heart and lungs. Journal of Applied Physiology, 106, 2060-2062. doi:10.1152/japplphysiol.90378.2008.

Masters, K. S., \& Ogles, B. M. (1998). Associative and dissociative cognitive strategies in exercise and cycling. 20 years later, what do we know? The Sport Psychologist, 12, 253-270.

Masters, R., \& Maxwell, J. (2008). The theory of reinvestment. International Review of Sport and Exercise Psychology, 1, 160-183. doi:10.1080/17509840802287218.

Morgan, W. P., \& Pollock, M. L. (1977). Psychological characteristics of elite cyclers. Annals of New York Academy of Science, 301, 382-403.

Noakes, T. D. (2012). Fatigue is a brain-derived emotion that regulates the exercise behaviour to ensure the protection of whole body homeostasis. Frontiers in Physiology, 3, 1-9. 
1 Perry, F. D. (2012). Biofeedback \& neurofeedback applications in sport psychology. The Sport Psychologist, 26, 313-314.

Raglin, J. S., \& Wilson, G. S. (2008). Psychology in endurance performance. In R. J. Shephard \& P. O. Astrand (2nd ed., pp. 211-219 The encyclopaedia of sports medicine. New York, NY: John Wiley \& Sons.

Razon, S., Basevitch, I., Filho, E., Land, W., Thompson, B., Biermann, M., \& Tenenbaum, G. (2010). Associative and dissociative imagery effects on perceived exertion, attention, and task duration. Journal of Imagery Research in Sport and Physical Activity, 5, 125.

Robazza, C., Pellizzari, M., Bertollo, M., \& Hanin, Y. L. (2008). Functional impact of emotions on athletic performance: Comparing the IZOF model and the directional perception approach. Journal of Sports Sciences, 26, 1033-1047. doi:10.1080/02640410802027352.

Russell, W. D., \& Weeks, D. L. (1994). Attentional style in ratings of perceived exertion during physical exercise. Perceptual and Motor Skills, 78, 779-783. doi:10.2466/pms.1994.78.3.779.

Russell, J. A., Weiss, A., \& Mendelsohn, G. A. (1989). Affect grid: A single-item scale of pleasure and arousal. Journal of Personality and Social psychology, 57, 493. doi:10.1037/0022-3514.57.3.493.

Schomer, H. H. (1986). Mental strategy and the perception of effort of marathon runners. International Journal of Sport Psychology, 17, 41-59.

Schücker, L., Anheier, W., Hagemann, N., Strauss, B., \& Völker, K. (2013). On the optimal focus of attention for efficient running at high intensity. Sport, Exercise, and Performance Psychology, 2, 207-219. doi:10.1037/a0031959. 
1 Stanley, C. T., Pargman, D., \& Tenenbaum, G. (2007). The effect of attentional coping strategies on perceived exertion in a cycling task. Journal of Applied Sport Psychology, 19, 352-363. doi:10.1080/10413200701345403.

Stevinson, C. D., \& Biddle, S. J. (1998). Cognitive orientations in marathon running and "hitting the wall”. British Journal of Sports Medicine, 32, 229-234. doi:10.1136/bjsm.32.3.229.

Tenenbaum, G. (2001). A social-cognitive perspective of perceived exertion and exertion tolerance. In R. N. Singer, H. A. Hausenblas, \& C. Janelle (Eds.), Handbook of sport psychology (pp. 810-822). New York: Wiley.

Tenenbaum, G. (2005). The study of perceived and sustained effort: concepts, research findings, and future directions. In D. Hackfort, J. Duda, \& R. Lidor (Eds.), Handbook of research on applied sport psychology (pp. 335-349) Morgantown, WV: Fitness Information Technology.

Wasserman, K., Stringer, W. W., Casaburi, R., Koike A., \& Cooper, C. B. (1994). Determination of the anaerobic threshold by gas exchange: Biochemical considerations, methodology and physiological effects. Zeitschrift für Kardiologie, 83, 1-12.

Weinberg, R. S., Smith, J., Jackson, A., \& Gould, D. (1984). Effects of association, dissociation, and positive self-talk strategies on endurance performance. Canadian Journal of Applied Sport Science, 9, 25-32. 
1 Table 1

2 Descriptive Statistics of Physiological, Performance, and Affective Data

\begin{tabular}{|c|c|c|}
\hline Variable & $M$ & $S D$ \\
\hline VT2- $\mathrm{VO}_{2}\left(\mathrm{~mL} \mathrm{~kg}^{-1} \mathrm{~m}^{-1}\right)$ & 23.08 & 9.88 \\
\hline VT2-Power (Watt) & 138.82 & 54.84 \\
\hline VT2-HR & 142.06 & 24.74 \\
\hline PPR (rpm) & 75.41 & 15.06 \\
\hline \multicolumn{3}{|l|}{ Time to exhaustion (min) } \\
\hline Type 1 performance & 18.35 & 6.93 \\
\hline Type 2 performance & 17.65 & 6.52 \\
\hline Type 3 performance & 14.12 & 5.95 \\
\hline \multicolumn{3}{|l|}{ Arousal } \\
\hline Type 1 performance & 5.44 & 1.89 \\
\hline Type 2 performance & 5.90 & 1.70 \\
\hline Type 3 performance & 5.56 & 1.90 \\
\hline \multicolumn{3}{|l|}{ Hedonic tone } \\
\hline Type 1 performance & 5.25 & 1.88 \\
\hline Type 2 performance & 5.33 & 1.72 \\
\hline Type 3 performance & 4.87 & 1.59 \\
\hline
\end{tabular}

3 Note. VT2 = second ventilatory threshold; $\dot{\mathrm{V}} \mathrm{O}_{2}=$ oxygen consumption; $\mathrm{HR}=$ heart rate; $\mathrm{PPR}$ $4 \quad$ = preferred pedaling rate. 